\title{
INCENTIVE EFFECTS OF GOVERNMENT MANDATED COST SHIFTING
}

\author{
Amporfu, Eugenia \\ Department of Economics, KNUST, Kumasi, Ghana, \\ Email: eamporfu@gmail.com
}

\begin{abstract}
This theoretical paper considers mandated cost shifting as a way of providing care for the poor. The method is similar to user fee health care systems with exemption policies for the poor. In the model, the government mandates the proper treatment of illness regardless of the ability to pay, and enforces that mandate with investigation. The results show that under costly investigation the physician randomly cheats by providing the wrong treatment to some types of patients. In response, the government also randomly investigates the treatment of such patients. The results also showed that cost shifting, hence user fee, deteriorates the welfare of both the rich and the poor as investigation becomes costly.
\end{abstract}

Keywords: costliness of government; mandated cost shifting; cheating; investigation.

\section{INTRODUCTION}

In every society there are poor individuals. A policy issue is the provision of the necessities of life to the poorest individuals in a society. One necessity is health care. If a society is not willing to see the poor die from treatable diseases then the provision of health care must involve some component of redistribution from the rich to the poor. Even with improvements in heath-care technology, this is a problem, which may get worse. As health-care technology improves, the cost of treating some diseases may fall, but the set of treatable diseases may grow.

One contribution mechanism that is common in the health care market of many developing countries is user fees. User fee does not involve redistribution from the rich to the poor. However, it is not socially desirable for the poor to be denied treatment as a result of inability to pay. Thus most user fee policies have exemption policies for the poor in society. According to the literature (e.g., Gilson, 1997; Nyonator and Kutzin, 1999; Valdivia, 2005; Haveman, 2009), the difficulty of identifying the poor makes the 


\section{Government Mandated Cost Shifting}

exemption policies non-functional in practice (the policy may still be non-functional in small communities where the poor can easily be identified). The implication here is that the rich receive the treatment they desire and the poor go untreated. This may not always be the case because both private and public health facilities may not want to be notorious about denying care to the poor. Health care providers may then find it desirable to provide care to the poor and charge it to the rich. This paper shows the welfare implications of such behaviour on both the rich and the poor.

The paper models a system in which the government mandates health care providers to shift cost from poor patients to rich patients. The exemption policies in the health care system with user fee are designed with the intention that access to life-saving treatment would not depend on ability to pay. A question is, do people die from treatable diseases due to an inability to pay in these societies? The answer is yes, but fewer than one might initially imagine. In Ghana for example mission hospitals (these are hospitals owned by Islam or Christian churches) would not send uninsured patients away as a result of inability to pay but would but would keep them in the hospitals until the fee is paid. Undoubtedly, many such patients would be allowed to go home when it becomes obvious that payment is impossible. Such practice is even found in public hospitals in developed economies as well. Take the case of Canada prior to the implementation of a publicly financed health care system. Consider a community doctor faced by a patient with a lifethreatening disease that could be treated but only at a cost beyond the means of the patient. Were these patients allowed to die? Undoubtedly some were, but some doctors treated the patients, billed them, but did not collect payment (Evans, 1984). How would a doctor or a hospital cover cost with revenue below costs for poor patients? The answer, of course, is with revenue above costs for rich patients, that is, with cost shifting. It is a form of private redistribution from rich to poor or from orchestrated by doctors and hospitals. The redistribution can also be from patients with adequate insurance to those with inadequate insurance (McArdle, 2009; Peduda, 2006).

Non-user fee health care systems, such as national health insurance system in Ghana, publicly financed health care in Canada, subsidization of health care for the poor or aged in Kenya and Uganda, or even direct redistribution through the tax system as in the optimal taxation literature, are public-sector intensive approaches in comparison with cost-shifting. In particular under cost shifting, the redistribution from rich to poor is orchestrated by the doctor using the price system so that no resources flow through the hands of the government. If the operation of government uses resources (e.g. the taxman's salary) or wastes resources (e.g. corruption) then this lack of public sector intensity can be a comparative benefit of the cost-shifting approach. Government operation becomes even more costly in economies with large informal sector, as in the case of many developing countries.

The purpose of this paper is to study mandated cost-shifting as a redistributive approach to the problem of providing adequate health care to the poor in the presence of costly government. The costliness of government can be due to high cost of the resources used, corruption, mismanagement, etc. The model extends Leger (2000) by introducing two categories of patients, rich and poor. It combines the capitation in Leger with fee for services, and replaces the insurance firm in Leger with a costly government that mandates the treatment of patients regardless of ability to pay. The patient's illness is either of high 


\section{Government Mandated Cost Shifting}

or low severity, which can be treated with high or low treatment, respectively. Both patients pay the same fee for low treatment but the rich pay a higher fee for high treatment. Since mandated cost shifting allows physicians to charge the rich a higher price than the poor, it gives incentive to the selfish physician to cheat by providing inappropriate treatment to the patients. Government investigation is thus needed to give the physician the incentive to treat the patient appropriately. The physician pays a fine if found guilty, and government gives any excess revenue after investigation to the poor as a subsidy. The results of the study show that the physician has the incentive to cheat by over-providing care to the rich and under-providing care for the poor. Compared to the user fee case, the results indicate that even though society wants the poor to receive care regardless of ability to pay, the asymmetric information in health care provision (equivalent to costly investigation) leads to a welfare loss to both the rich and the poor.

\section{THE MODEL}

This is a game with two players: the physician (representing a health care provider and so can be a doctor, a hospital, a clinic, etc.,) and the government, and passive agents who are patients in a free entry and exit market. The use of free entry and exit allows the analysis to focus on the effect of mandated cost shifting without adding any other inefficiency that may exist in the health care market as a result of the absence of free entry and exit. There are two types of patients: rich and poor, indexed $j=R$ or $P$. The rich here includes middle income and above. Categorizing people into rich and poor is the practice even in the world of a continuum wealth. The United States for example, uses family income to determine whether one is (rich or middle income) or poor (and hence qualifies for Medicaid). In Ireland there are medical card patients (poor) and private patients (rich or middle income). Let $r$ be the proportion of the rich among the patients and $(1-r)$ be the proportion of the poor. A patient gets sick only once, drawing from two severities of illness: low severity $\left(\theta^{L}\right)$ and high severity $\left.\theta^{H}\right)$. The rich and the poor draw from the distribution $\theta=\left\{\theta^{L}, \theta^{H}\right\}$ with probabilities $\pi_{j}$ and (1- $\left.\pi_{j}\right)$ respectively. The successful treatment of $\theta^{H}$ requires high treatment, $e^{H}$, and that of $\theta^{L}$ requires low treatment, $e^{L}$, with $e^{L}<e^{H}$. Income is observable to the doctor and the government. In developing countries where there is a large informal sector peoples' income are identified by their area of residence. Two cases are examined: costless investigation and costly investigation. Both the physician and the government can costlessly observe the type of illness under costless investigation but only the physician observes the type of illness costlessly with costly investigation. After observing the type of illness, the physician chooses the treatment type. While the illness type is not costlessly observable to the government, the treatment type is observable.

The government collects a lump-sum tax, $\gamma_{R}$, from rich patients to fund the investigation of the physician and for redistribution to the poor. It pays a lump-sum subsidy, $\gamma_{p}$, to the poor. In an extreme case in which it is too costly for the government to collect any revenue, it can borrow to finance investigation. A patient pays a fee, $C^{i}(i=H$ or $L)$, for treatment and spends the rest of their income, $y_{j \pm} \gamma_{j^{-}} C^{i}$, on all other goods and services consumed, where $y_{j}-\gamma_{j}=y_{R}-\gamma_{R}$ for $j=R$ and $y_{j}+\gamma_{j}=y_{p}+\gamma_{p}$. Their health status, $\delta\left(\theta^{i}, e^{i}\right)$, is a function of illness and treatment. Their utility function is: $U_{j}\left(y_{j} \pm \gamma_{j}-C^{i}, \delta\left(\theta^{i}, e^{i}\right)\right)$, and $U_{l}>0$, 


\section{Government Mandated Cost Shifting}

$U_{2}>0$ and $U_{11} \leq 0, U_{21}=U_{12}=0$. The physician's utility is $V\left(C^{i}, e^{i}\right) ; V_{2}<0, V_{1}>0, V_{11}<$ 0 , and $V(0,0)=0$. The assumption $U_{12}=0$ is added to simplify the calculations. The removal of this assumption does not affect the results qualitatively.

In a free entry and exit market, physicians earn zero profit for each type of treatment and so $V\left(C^{L^{*}}, e^{L}\right)=V\left(C^{H^{*}}, e^{H}\right)=0$ and with $e^{H}>e^{L}$, it follows that $C^{H^{*}}>C^{L^{*}}$. In a free entry and exit market $V\left(C^{H^{*}}, e^{H}\right)=V\left(C^{L^{*}}, e^{L}\right)=0$ because if one is positive the physician increase their utility by providing the treatment type that provides positive utility. Besides with free entry and exit, other physicians will enter and compete it away. Alternatively, if one is negative then physicians will exit the market. Without any redistribution it is assumed that the poor cannot afford $C^{H^{*}}$. With $\gamma_{p}=0$, it is also assumed the poor cannot pay more than $C^{L^{*}}$ for health care. It is assumed that the poor cannot afford the actuarially fair insurance premium: $\pi_{p} C^{L}+\left(1-\pi_{p}\right) C^{H}$. A standard approach to such inequality would be for the government to redistribute income through the tax system by collecting enough revenue to subsidise the poor who receive high treatment. However, such a solution is not necessarily optimal in this model because of the additional assumption of a costly government. Following Burbridge and Myers (2004), the model assumes that government is costly. The assumption is that for every dollar of revenue collected a given percentage, $\omega$, is lost. In an extreme case in which $\omega=1$ all revenue collected is lost through the costliness of government.

If the government uses a tax system then the government's budget is balanced if what is collected after waste is equal to what the poor with high treatment receive, i.e., $r(1-\omega) \gamma_{R}$ $=(1-r)\left(1-\pi_{p}\right)\left(C^{H^{*}}-C^{L}\right)$, which implies that $\gamma_{R}=\frac{(1-r)\left(1-\pi_{p}\right)\left(C^{H^{*}}-C^{L}\right)}{r(1-\omega)}$. Thus $\gamma_{R}$ approaches infinity as $\omega$ approaches one, for any finite $\left(C^{H^{*}}-C^{L}\right)$. The use of the tax system for redistribution in the presence of a costly government then is not optimal.

Alternatively, the government is allowed to redistribute income through the health care system by directly mandating the physician to give any patient the proper treatment and to charge the poor $C^{L^{*}}$ for either treatments, and charge the rich $C^{L^{*}}$ for $\mathrm{e}^{L}$. The market then determines the high treatment fee, $C^{S}$, paid by the rich through free entry and exit of physicians or zero expected profit.

With both the rich and the poor paying $C^{L^{*}}$ for low treatment such that $V\left(C^{L^{*}}, e^{L}\right)=$ 0 , with $e^{H}>e^{L}$, and with the poor paying $C^{L^{*}}$ for $e^{H}$, it follows that $V\left(C^{L^{*}}, e^{H}\right)<0$, i.e., the physician makes a loss from treating a poor patient with $e^{H}$. The physician can only earn a positive utility if $V\left(C^{S^{*}}, e^{H}\right)>V\left(C^{H^{*}}, e^{H}\right)=0$ implying that $C^{S^{*}}>C^{H^{*}}>C^{L^{*}}$. The focus of the paper is redistribution not insurance. The poor patient pays $C^{L^{*}}$ in either illness state so has no incentive to insure. The rich patient can be assumed risk neutral to avoid the insurance issue. The inequality however creates incentives for the physician to cheat when choosing treatment type. The physician may not always use the right type of treatment, but here the choice of treatment would depend on the patient's income type. It is assumed then that with probability $\alpha_{j}^{L / H}$ the physician chooses $e^{L}$ to treat a patient of type $j$, given that the patient has drawn $\theta^{H}$ (cheats), and with probability $\left(1-\alpha_{j}^{L / H}\right)$ she treats the patient with $e^{H}$. With probability $\alpha_{j}^{L / L}$ the physician chooses $e^{L}$ to treat a patient 
who has drawn $\theta^{L}$ and with a probability $\left(1-\alpha_{j}^{L / L}\right)$ the physician treats the patient with $e^{H}$ (cheats).

In order to protect the welfare of patients, the government investigates the physician. The timing is as follows. The government mandates the following behaviour from the physician: for all patients, treat $\theta^{H}$ with $e^{H}$ and charge the poor $C^{L^{*}}$, treat $\theta^{L}$ with $e^{L}$ and charge $C^{L^{*}}$ whether the patient is rich or poor. Next, a patient of an income type comes to a doctor and must be accepted. This is a strong assumption but a structure where doctors are expected to do some treatment for the poor could be constructed. One can consider a case in which society frowns at doctors who send poor patients away without treatment. The need for a good reputation (in order not to lose patients) then can induce doctors to provide some treatment even if inadequate regardless of ability to pay. The income type is observable to both the doctor and the government. (In a developed economy where the formal sector is large one can imagine the patient's ID card, issued by the government, reflects income type. In a developing economy where the informal sector is large, patient's income type could be identified by their address or race). The doctor costlessly observes the illness type. The doctor then chooses whether or not to cheat $(\alpha)$. Simultaneously, the government chooses whether or not to investigate conditional on the observed income type and treatment type $e^{H}$ or $e^{L}$. Because the patient's illness type is not observable to the government without investigation, there is private information and the solution concept is Bayesian Nash equilibrium. Thus, it is assumed that the government investigates the physician with probability $\mu_{j}^{L}$ for a patient of income type $j$ who has received $e^{L}$, and with probability $\left(1-\mu_{j}^{L}\right)$ it does not investigate. For $e^{H}$, it investigates with probability $\mu_{j}^{H}$, and with $\left(1-\mu_{j}^{H}\right)$ it does not investigate.

If the government investigates it will be able to find out whether or not the physician used the right treatment, but it incurs a cost, $k$. If the physician is found guilty of using the wrong effort she pays a fine, $\phi$. As discussed in Becker (1968) and Shavell (1991), setting the fine sufficiently high would deter the physician from cheating. The problem with this is that of credibility (Andreoni, 1991). If the government sets the fine extremely high then the physician will not expect the government to implement it and so will cheat. Thus it is only assumed that the fine, $\phi$, satisfies the conditions which require that the physician is better off not cheating than cheating and being caught with certainty (One advantage of cost shifting is that this usual credibility issue is less of a problem here because the physician who does not treat a severely ill patient, for financial reason, could expect a serious punishment):

$$
\begin{aligned}
0>V\left(C^{L^{*}}, e^{H}\right) & >V\left(C^{L^{*}}-\phi, e^{L}\right) \\
0 & =V\left(C^{L^{*}}, e^{L}\right)>V\left(C^{S^{*}}-\phi, e^{H}\right)
\end{aligned}
$$

Equation (1) states that given the government investigates, the fine is high enough such that the physician is better off using $e^{H}$ to treat a poor patient who has drawn $\theta^{H}$, than treating him with $e^{L}$ and being fined. Similarly, (2) states that, given the government investigates, the fine is high enough such that the physician is better off using $e^{L}$ to treat a rich patient who has drawn $\theta^{L}$, than treating him with $e^{H}$ and being fined.

As already explained, it costs resources for the government to collect taxes. Given that a physician is found guilty, the physician pays a fine. The fine collection is assumed to require less time resource than that required to locate a rich person and tax the one. Let $\sigma$ 


\section{Government Mandated Cost Shifting}

denote the extent to which the cost of tax collection exceeds the cost of fine collection. Thus the costliness of government for fine collection is $\tau=\omega-\sigma$. The following assumptions are added:

Assumption 1: $U\left(y_{j} \pm \gamma_{j}-C^{g}, \delta\left(\theta^{H}, e^{H}\right)\right)>U\left(y_{j} \pm \gamma_{j}-C^{g}, \delta\left(\theta^{H}, e^{L}\right)\right),(g=S, L)$ a patient of type $j$ with high severity of illness is better off when treated with $e^{H}$ than when treated with $e^{L}$ given the fees for type $j$.

Assumption 2: $U\left(y_{j} \pm \gamma_{j}-C^{L}, \delta\left(\theta^{L}, e^{L}\right)\right)>U\left(y_{j} \pm \gamma_{j}-C^{g}, \delta\left(\theta^{L}, e^{H}\right)\right)$, a patient with low severity of illness is better off when treated with $e^{L}$ than when treated with $e^{H}$ given the fee paid.

Assumption 3: $k<\left(1-\pi_{p}\right)(1-\tau) \phi$ and $k<\pi_{R}(1-\tau) \phi$. The expected net fine collected from investigation, given the physician is found guilty, exceeds the cost of investigation. This assumption implies that the costliness of government for fine collection is strictly less than one in the model. It is shown later on in the paper that when the government is costly enough to make mandated cost shifting more optimal than the direct redistribution of tax.

It is assumed that and the government's objective function, $E W\left(\alpha_{j}^{L / L}, \alpha_{j}^{L / H}, \gamma_{j}\right)$, is the sum of the expected utilities of the patients:

$$
\begin{aligned}
& E \\
& \quad+\left(\alpha_{j}^{L / L}, \alpha_{j}^{L / H}, \gamma_{j}\right)=(1-r)\left[\left(1-\beta_{P}^{L}\right) U_{p}\left(y_{p}+\gamma_{p}-C^{L}, \delta\left(\theta^{H}, e^{L}\right)\right)\right. \\
& \quad+\beta_{P}^{L} U_{p}\left(y_{p}+\gamma_{p}-C^{L}, \delta\left(\theta^{L}, e^{L}\right)\right) \\
& \left.\quad+\beta_{P}^{H} U_{p}\left(y_{p}+\gamma_{p}-C^{L}, \delta\left(\theta^{H}, e^{H}\right)\right)+\left(1-\beta_{P}^{H}\right) U_{p}\left(y_{p}+\gamma_{p}-C^{L}, \delta\left(\theta^{L}, e^{H}\right)\right)\right] \\
& \quad+r\left[\beta_{R}^{L} U_{R}\left(y_{R}-\gamma_{R}-C^{L}, \delta\left(\theta^{L}, e^{L}\right)\right)+\left(1-\beta_{R}^{L}\right) U_{R}\left(y_{R}-\gamma_{R}-C^{L}, \delta\left(\theta^{H}, e^{L}\right)\right)\right. \\
& \left.\quad+\beta_{R}^{H} U_{R}\left(y_{R}-\gamma_{R}-C^{S}, \delta\left(\theta^{H}, e^{H}\right)\right)+\left(1-\beta_{R}^{H}\right) U_{R}\left(y_{R}-\gamma_{R}-C^{S}, \delta\left(\theta^{L}, e^{H}\right)\right)\right]
\end{aligned}
$$

Where $\beta_{j}^{L^{*}}=\frac{\pi_{j} \alpha_{j}^{L / L^{*}}}{\left(1-\pi_{j}\right) \alpha_{j}^{L / H^{*}}+\pi_{j} \alpha_{j}^{L / L^{*}}}, \beta_{j}^{H^{*}}=\frac{\pi_{j} \alpha_{j}^{H / L^{*}}}{\left(1-\pi_{j}\right) \alpha_{j}^{H / H^{*}}+\pi_{j} \alpha_{j}^{H / L^{*}}}$. The first two terms in the first square brackets represent a poor patient's expected utility when treated with $e^{L}$. The second two terms represent the patient's expected utility when treated with $e^{H}$. Similarly, the first two terms in the second square brackets represent the rich patient's expected utility when treated with $e^{L}$ and the remaining two terms represent his expected utility when treated with $e^{H}$. The government's budget constraint is:

$$
\begin{aligned}
& r(1-\omega) \gamma_{R}-(1-r) \gamma_{P}+(1-r)\left[\beta_{P}^{L} \mu_{P}^{L}(-k)+\left(1-\beta_{P}^{L}\right) \mu_{P}^{L}((1-\tau) \phi-k)\right. \\
& \left.+\beta_{P}^{H} \mu_{P}^{H}(-k)+\left(1-\beta_{P}^{H}\right) \mu_{P}^{H}((1-\tau) \phi-k)\right]+r\left[\beta_{R}^{H} \mu_{R}^{H}(-k)+\left(1-\beta_{R}^{H}\right) \mu_{R}^{H}((1-\tau) \phi-k)\right. \\
& \left.\quad+\beta_{R}^{L} \mu_{R}^{L}(-k)+\left(1-\beta_{R}^{L}\right) \mu_{R}^{L}((1-\tau) \phi-k)\right]=0
\end{aligned}
$$

The first term in (4) represents the lump-sum tax collected from the rich after waste and the second term represents the lump-sum subsidy that is transferred to the poor. The first two terms in the first square bracket represent the expected net fine collected from investigating the physician for treating a poor patient with $e^{L}$. The two remaining terms represent the expected fine collected from investigating the physician for treating the poor with $e^{H}$. The second square bracket represents expected fine collected from investigating the physician for the treatments provided to the rich with the first two terms 
for the high treatment and the last two for low treatment. The physician's expected utility if faced by a poor patient is:

$$
\begin{aligned}
& E V\left(\alpha_{j}^{L / H}, \alpha_{j}^{L / L}, \mu_{j}^{i}\right)=\left(1-\pi_{P}\right)\left[\left(1-\alpha_{P}^{L / H}\right) V\left(C^{L}, e^{H}\right)\right. \\
& \left.\quad+\alpha_{P}^{L / H}\left(\mu_{P}^{L} V\left(C^{L}-\phi, e^{L}\right)+\left(1-\mu_{P}^{L}\right) V\left(C^{L}, e^{L}\right)\right)\right] \\
& \quad+\pi_{P}\left[\alpha_{P}^{L / L} V\left(C^{L}, e^{L}\right)+\left(1-\alpha_{P}^{L / L}\right)\left(\mu_{P}^{H} V\left(C^{L}-\phi, e^{H}+\left(1-\mu_{P}^{H}\right) V\left(C^{L}, e^{H}\right)\right)\right]\right.
\end{aligned}
$$

The first square bracket in the first term represents the expected utility from treating a poor patient with $e^{L}$. The second square bracket represents the expected utility from treating the poor patient with $e^{H}$.

If faced by a rich patient the physician's expected utility is:

$$
\begin{aligned}
& E V\left(\alpha_{j}^{L / H}, \alpha_{j}^{L / L}, \mu_{j}^{i}\right)=\left\{\pi_{R}\left[\left(1-\alpha_{R}^{L / L}\right)\left(\mu_{R}^{H} V\left(C^{S}-\phi, e^{H}\right)+\left(1-\mu_{R}^{H}\right) V\left(C^{S}, e^{H}\right)\right)+\alpha_{R}^{L / L} V\left(C^{L}, e^{L}\right)\right]\right. \\
& \quad+\left(1-\pi_{R}\right)\left[\alpha_{R}^{L / H}\left(\mu_{R}^{L} V\left(C^{L}-\phi, e^{L}\right)+\left(1-\mu_{R}^{L}\right) V\left(C^{L}, e^{L}\right)\right)+\left(1-\alpha_{R}^{L / H}\right) V\left(C^{S}, e^{H}\right)\right]
\end{aligned}
$$

The first square bracket represents the doctor's expected utility from treating a rich patient with $e^{H}$ and the second square bracket represents her expected utility from treating a rich patient with $e^{L}$. The game is between a doctor with one patient and the government. But it can be generalized to more than one patient, $N$, by assuming enough linearity in the doctor's utility function or $N V(C, e)=V\left(N C^{i}, N e^{i}\right)$. Example, $V(C, e)=C^{i}-e^{i}$. The model is solved and discussed under two main cases: costless and costly investigation cases.

\section{COSTLESS INVESTIGATION CASE}

Assume the government does not incur any cost of investigation, $k=0$. The government chooses its strategies to maximize (3) subject to (4) with $k=0$. Its strategies consist of choosing $\mu_{j}^{i}$ taking $\alpha_{j}^{i}$ and $C^{S^{*}}$ as given and choosing the optimal tax and subsidy. Notice that (3) is not a function of $\mu_{j}^{i}$ so the investigation is for revenue reasons only. The physician simultaneously chooses treatment type after observing the illness type, taking $\mu_{j}^{i}$ as given. The Bayesian Nash equilibrium strategies are as follows:

$$
\begin{aligned}
& 1>\mu_{p}^{H^{*}} \geq 0, \quad 1>\mu_{R}^{L^{*}} \geq 0, \quad \mu_{P}^{L^{*}} \geq \bar{\mu}_{P}^{L^{*}} \equiv \frac{V\left(C^{L^{*}}, e^{H}\right)}{V\left(C^{L^{*}}-\phi, e^{L}\right)} \\
& \mu_{R}^{H^{*}} \geq \bar{\mu}_{R}^{H^{*}} \equiv \frac{V\left(C^{S^{*}}, e^{H}\right)}{V\left(C^{S^{*}}, e^{H}\right)-V\left(C^{S^{*}}-\phi, e^{H}\right)}
\end{aligned}
$$

PROPOSITION 1: Given that $k=0$, the government investigates with probability less than one when it observes that a rich patient has received a low treatment or a poor patient has received a high treatment. However, the government may always investigate or investigates with a high probability when it observes that a rich patient has received a high treatment or a poor patient has received a low treatment. (Proof: see appendix 1.3 a)

From (1) and (2), $0<\bar{\mu}_{R}^{L^{*}}<1,0<\bar{\mu}_{R}^{H^{*}}<1$. Intuitively, with $V\left(C^{S^{*}}, e^{H}\right)>V\left(C^{L^{*}}, e^{L}\right)$, the physician has incentive to cheat when treating a rich patient who has low severity of illness and a poor patient who has high severity of illness. Thus, the government will 
always investigate or investigate at a high probability because it incurs no cost in doing so and that it will collect a fine if the physician is found guilty. However, with $V\left(C^{L^{*}}, e^{H}\right)$ $<V\left(C^{L^{*}}, e^{L}\right)$, the physician has no incentive to treat a rich patient who has high severity of illness with low treatment or a poor patient who has low severity of illness with high treatment. However, because investigation is costless, the government may investigate when it observes that a poor patient has been treated with high treatment and a rich patient with low treatment, i.e., $1>\mu_{p}^{H^{*}} \geq 0,1>\mu_{R}^{L^{*}} \geq 0$.

The physician chooses her strategies to maximize her expected utility taking the government's strategies as given. The strategies are as follows:

$$
\alpha_{p}^{L / H^{*}}=\left(1-\alpha_{p}^{L / L^{*}}\right)=\left(1-\alpha_{R}^{L / L^{*}}\right)=\alpha_{R}^{L / H^{*}}=0,
$$

PROPOSITION 2: Given $k=0$, the physician plays the pure strategy of not cheating when treating patients. (Proof: see appendix 1.3.a).

Intuitively, with $V\left(C^{S^{*}}, e^{H}\right)>V\left(C^{L^{*}}, e^{L}\right)$, the physician has no incentive to treat a rich patient who has high severity of illness with low treatment, hence $\alpha_{R}^{L / H^{*}}=0$. In the same way, with $V\left(C^{L^{*}}, e^{H}\right)<V\left(C^{L^{*}}, e^{L}\right)$, the physician has no incentive to treat a poor patient who has low severity of illness with high treatment, hence $\alpha_{p}^{L / L^{*}}=1$. If the government finds the physician has used a wrong effort it fines the physician. The physician, knowing that the government can observe the type of illness costlessly, expects to pay a fine anytime she uses the wrong effort to treat a patient. By (1) and (2), the physician is better off providing the right treatment than providing a wrong treatment and being fined. Thus, the physician will always provide the right effort. With $k=0$, the government does not need to collect revenue for investigation so $\gamma_{R}{ }^{*}=\gamma_{p}{ }^{*}=0$.

The equilibrium is efficient or first best because there is no cheating and no costly investigation or taxation. Both patients receive the right treatment and the rich do not have to pay for investigation through the tax system. Assuming that each doctor's draw of patients out of the rich/poor patient distribution is that of the population $(r, 1-r)$ and that the doctor's utility function satisfies $N V\left(C^{i}, e^{i}\right)=V\left(N C^{i}, N e^{i}\right)$. Substituting in (7), $C^{S^{*}}$ can be determined by setting the physician's expected utility to zero (free entry and exit):

$$
r\left(1-\pi_{R}\right) V\left(C^{S^{*}} e^{H}\right)+(1-r)\left(1-\pi_{P}\right) V\left(C^{L^{*}} e^{H}\right)=0
$$

This determines $C^{S^{*}}\left(C^{L}, \pi_{R}, \pi_{p}, r, e^{H}\right)$. These results are driven by the costless investigation on the type of illness. The assumption of $k>0$ will give second-best results.

\section{COSTLY INVESTIGATION CASE}

The government can find the illness type but only through investigation at cost $k>0$. As before the government chooses $\mu_{j}^{i}, \in(0,1)$, and $\gamma_{j}$, taking $\alpha_{j}^{i}$ as given, to maximize (3) subject to (4). Similarly, the physician simultaneously chooses $\alpha_{j}^{L / L}, \in(0,1)$, and $\alpha_{j}^{L / H} \in$ $(0,1)$, taking $\mu_{j}^{i}$ as given, to maximize (5). The government's equilibrium strategies are as follows:

$$
\begin{aligned}
& \mu_{p}^{H^{*}}=\mu_{R}^{L^{*}}=0 \\
& \mu_{P}^{L^{*}}=\bar{\mu}_{P}^{L^{*}}, \quad \mu_{R}^{H^{*}}=\bar{\mu}_{R}^{H^{*}}
\end{aligned}
$$


where $\bar{\mu}_{P}^{L^{*}}$ and $\bar{\mu}_{R}^{H^{*}}$ are defined in (6).

PROPOSTION 3: The government investigates the physician with probabilities that are strictly positive and less than one when it observes the physician has provided high treatment for a rich patient and low treatment for a poor patient. However the government does not investigate when it observes a poor patient is treated with high treatment and when a rich patient is treated with low treatment. (Proof: see appendix 1.3.b).

From (1) and (2) $\mu_{p}^{L^{*}}$ and $\mu_{R}^{H^{*}}$ are positive and less than one and so are mixed strategies. The government plays the pure strategy of not investigating when it observes that a poor patient has received high treatment and a rich patient has received low treatment. The government however plays a mixed strategy when it observes that a poor patient has received low treatment and a rich patient has received high treatment.

Intuitively, $\mu_{R}{ }^{L^{*}}=0$ because with $V\left(C^{S^{*}}, e^{H}\right)>V\left(C^{L^{*}}, e^{L}\right)$, the physician has no incentive to treat a rich patient with $e^{L}$ given that the patient has drawn $\theta^{H}$. Similarly, $\mu_{p}{ }^{H^{*}}=0$, i.e., the government does not investigate when it observes that a poor patient has been treated with $e^{H}$. As before, this is because with $V\left(C^{L^{*}}, e^{L}\right)>V\left(C^{L^{*}}, e^{H}\right)$, the physician has no incentive to use $e^{H}$ to treat a poor patient given that the patient has drawn $\theta^{L}$. However, with $V\left(C^{S^{*}}, e^{H}\right)>V\left(C^{L^{*}}, e^{L}\right)$, the physician has the incentive to treat a rich patient with $e^{H}$ given that the patient has drawn $\theta^{L}$. In the same way, with $V\left(C^{L^{*}}, e^{L}\right)>V\left(C^{L^{*}}, e^{H}\right)$ the physician has the incentive to treat a poor patient with $e^{L}$ given that the patient has drawn $\theta^{H}$. If the government always investigates the physician will never cheat. But if the physician never cheats then the government will not investigate. Thus, the government plays a mixed strategy in equilibrium.

The physician's strategies are:

$\alpha_{p}^{L / L^{*}}=1, \alpha_{R}^{L / H^{*}}=0, \alpha_{p}^{L / H^{*}}=\frac{\pi_{p} k}{\left(1-\pi_{p}\right)((1-\tau) \phi-k)}, \quad\left(1-\alpha_{R}^{L / L^{*}}\right)=\frac{\left(1-\pi_{R}\right) k}{\pi_{R}((1-\tau) \phi-k)}$

PROPOSITION 4: With probabilities that are positive but less than one, the physician provides high treatment to a rich patient given that he has drawn low severity of illness and provides low treatment to a poor patient given that he has drawn high severity of illness. However, the physician always provides low treatment to a poor patient given that the patient has drawn low severity of illness and provides high treatment to a rich patient given that the patient has drawn high severity of illness. (Proof: see appendix 1.3.b).

Thus, $\alpha_{p}^{L / H^{*}}$ and $\left(1-\alpha_{R}^{L / L^{*}}\right)$ are positive and by Assumption 3 they are less than one. The physician again plays the pure strategy, of not cheating when treating a poor patient with low severity of illness and a rich patient with high severity of illness. The physician however plays a mixed strategy when treating a poor patient with high severity of illness or a rich patient with low severity of illness. Note that as $k$ approaches zero, the costless investigation results are obtained.

The assumption that it is too costly for government to collect revenue implies that $\gamma_{R}{ }^{*}=\gamma_{p}{ }^{*}=0$ (proof: see appendix 1.3.c). With free entry and exit, the physician's 


\section{Government Mandated Cost Shifting}

expected utility goes to zero in equilibrium. Then following the same procedure as used for (8) but now using (9) and (10), results in (8) again. Thus, $C^{S^{*}}\left(C^{L}, \pi_{R}, \pi_{p}, r, e^{H}\right)$ from $(\mathbf{8})$ is the same as that under costly investigation. This strong result comes from the mixed strategies. In equilibrium, the government chooses its strategies to make the physician indifferent between cheating and not cheating whether or not $k$ is zero.

The costly investigation equilibrium is Pareto inferior to the costless investigation equilibrium because those who get the right treatment are indifferent, but those who get the wrong treatment are worse off. The costly investigation case then is inefficient and the inefficiency consists of over-utilization of care for the rich and under-utilization of care for the poor.

The results so far show that as investigation becomes more costly the equilibrium of mandated cost shifting becomes less efficient. As already explained, the free entry and exit assumption gives the physician no incentive to cheat when there is no income difference between patients. Thus direct redistribution of income through the tax system should achieve efficiency in terms of treatment. Such equilibrium is achievable under mandated cost shifting if investigation is not costly. Efficiency then suggests that the government invest in innovative technologies to minimize the cost of investigation.

\section{CONCLUSION}

When it is too costly for a government to collect revenue, mandated cost shifting as a means of providing health services to the poor can be less wasteful than using the tax system. This paper has shown that, when investigation is costly, the physician randomly uses the wrong treatment for patients.

Even though government mandating direct redistribution of revenue from the rich to the poor is not observed in the real world, user fees can produce similar results in the real world. Because it is socially undesirable for the poor to be denied care, health care providers may be coerced into, at least sometimes, providing care to those who cannot pay. This can only be done through cost shifting which in turn creates incentive to sometimes provide the wrong treatment as a result of asymmetric information (equivalent to costly investigation).

Finally, the paper has assumed that information on the patient's income type is evenly distributed between the government and the physician. Efficiency can be distorted when the physician or patient has information advantage, because of the resulting increase in expected cost of investigation. Cost shifting then is more efficient when information on the patient's income is not costly to the government or physician.

\section{Acknowledgements}

This paper is based on a chapter of the author's $\mathrm{PhD}$ thesis. The author would like to thank Gordon Myers, Nicolas Schmit, J. Atsu Amegashie and Steve Mongraine for their valuable comments and contributions. The author also thanks members of the brown bag seminar at the economics department of Simon Fraser University. 


\section{References}

Andreoni, J. (1991). "Reasonable doubt and the optimal magnitude of fines: should the penalty fit the crime?" Rand Journal of Economics 22: 385-95.

Becker, G.S., (1968). "Crime and punishment: An economic approach,” Journal of Political Economy 76: 169-217.

Burbridge, J.B. and Myers, G.M. (2004). "Tariff wars and trade deals with costly government," Review of International Economics 12: 543-549.

Butler, R.J, Hartwig, R.P. and Gardner, H. (1997). "HMOs, moral hazard and cost shifting in workers' compensation," Journal of Health Economics 16: 191-206.

Evans, R.G. (1984). Strained Mercy: The Economics of Canadian Health Care, Toronto: Butterworths.

Gertler, P.J. (1989). "Subsidies, quality, and the regulation of nursing homes," Journal of Public Economics 38: 33-52.

Gilson, L. (1997). "The lessons of user fee experience in Africa," Health Policy and Planning 12:273-285.

Haveman, R. (2009). "What does it mean to be poor in a rich society?" Focus 26(2): 8186.

Lee, J.A., H. Birnbaum, H., and C. Bishop (1983). "How nursing homes behave: A multi-equation model nursing home behaviour," Social Science Med. 17: 18971906.

Leger, P. T. (2000). "Quality control mechanisms under capitation payment for medical services," Canadian Journal of Economics 33(2): 565-86.

McArdle, M. (2009), "Further thoughts on hospital cost shifting," The Atlantic, http://www.theatlantic.com/business/archive/2009/10/further-thoughts-on-hospital-costshifting/28522/

Norton, E. (1992). "Incentive regulation of nursing homes," Journal of Health Economics 11: 105-28.

Nyonator, F. and Kutzin J. (1999). "Health for some? The effects of user fees in the Volta Region of Ghana," Health Policy and Planning, 14(4): 329-341.

Paduda, J. (2006). "Cost shifting to private payers," Managed Care Matters, http://www.joepaduda.com/archives/000380.html

Pauly, M. (1981). Doctors and their Workshops, U. of Chicago Press, Chicago.

Shavell, S. (1991). "Specific versus general enforcement of law," Journal of Political Economics, 99: 1088-1109.

Valdivia, M. (2005). "Peru: is identifying the poor the main problem 
in targeting nutritional programs" HNP Discussion Paper, Reaching the poor program no. 7 ,

http://siteresources.worldbank.org/HEALTHNUTRITIONANDPOPULATION/R esources/281627-1095698140167/RPP7ValdiviaPeruFinal.pdf

\section{Appendix: Proof of Propositions 1 - 4}

Deriving the strategies require working out the government's problem, the physician's problem, and the characterizing an equilibrium. Subsection 1.1, focuses on the government's problem, 1.2 is the physician's problem and 1.3 characterizes equilibrium.

\subsection{The Governments Problem}

The Government's objective function $(E W)$ and budget constraint $(B C)$ are defined in (3) and (4) in the text. The $B C$ can be further simplified into

$$
\begin{aligned}
r(1- & \omega) \gamma_{R}-(1-r) \gamma_{P}+(1-r)\left[\mu_{P}^{L} \frac{\left(1-\pi_{p}\right) \alpha_{p}^{L / H}((1-\omega) \phi-k)-\pi_{p} \alpha_{p}^{L / L} k}{\left(1-\pi_{p}\right) \alpha_{p}^{L / H}+\pi_{p} \alpha_{p}^{L / L}}\right. \\
& \left.+\mu_{P}^{H} \frac{\pi_{P}\left(1-\alpha_{P}^{L / L}\right)((1-\omega) \phi-k)-\left(1-\pi_{P}\right)\left(1-\alpha_{P}^{L / H}\right) k}{\left(1-\pi_{P}\right)\left(1-\alpha_{P}^{L / H}\right)+\pi_{P}\left(1-\alpha_{P}^{L / L}\right)}\right] \\
& +r\left[\mu_{R}^{H} \frac{\pi_{R}\left(1-\alpha_{R}^{L / L}\right)((1-\omega) \phi-k)-\left(1-\pi_{R}\right)\left(1-\alpha_{R}^{L / H}\right) k}{\left(1-\pi_{R}\right)\left(1-\alpha_{R}^{L / H}\right)+\pi_{R}\left(1-\alpha_{R}^{L / L}\right)}\right. \\
& \left.+\mu_{R}^{L} \frac{\left(1-\pi_{R}\right) \alpha_{R}^{L / H}((1-\omega) \phi-k)-\pi_{R} \alpha_{R}^{L / L} k}{\left(1-\pi_{R}\right) \alpha_{R}^{L / H}+\pi_{R} \alpha_{R}^{L / L}}\right]=0
\end{aligned}
$$

And the non-negativity constraints $\mu_{p}{ }^{L} \geq 0, \mu_{p}{ }^{H} \geq 0, \mu_{R}{ }^{L} \geq 0, \mu_{R}{ }^{H} \geq 0, \gamma_{p} \geq 0, \gamma_{R} \geq 0$ and inequality constraints:

$$
\begin{array}{lll}
1-\mu_{p}^{L} \geq 0 & \text { (IE1) } & 1-\mu_{R}^{L} \geq 0 \\
1-\mu_{p}^{H} \geq 0 & \text { (IE2) } & 1-\mu_{R}^{H} \geq 0
\end{array}
$$

The government's problem is to maximize the Lagrangian

$$
\varphi=E W+\rho_{0} B C+\rho_{1} I E 1+\rho_{2} I E 2+\rho_{3} I E 3+\rho_{4} I E 4
$$

with the choices $\left\{\mu_{p}{ }^{L}, \mu_{p}{ }^{H}, \mu_{R}{ }^{L}, \mu_{R}^{H}, \gamma_{p}, \gamma_{R}\right\}$ taken as given $\left\{\alpha_{p}^{L / L}, \alpha_{p}^{L / H}, \alpha_{R}^{L / L}, \alpha_{R}{ }^{L / H}\right\}, C^{L^{*}}$ and $C^{S}$. Note that $E W$ is not a function of $\mu_{j}^{L}, \mu_{j}^{H}$. The partial derivatives are:

$$
\begin{aligned}
\frac{\partial \varphi}{\partial \mu_{p}^{L}} & =-\rho_{1}+(1-r) \rho_{0} \frac{\left(1-\pi_{p}\right) \alpha_{p}^{L / H}((1-\omega) \phi-k)-\pi_{p} \alpha_{p}^{L / L} k}{\left(1-\pi_{R}\right) \alpha_{p}^{L / H}+\pi_{R} \alpha_{p}^{L / L}} \\
\frac{\partial \varphi}{\partial \mu_{p}^{H}} & =-\rho_{2}+(1-r) \rho_{0} \frac{\pi_{P}\left(1-\alpha_{P}^{L / L}\right)((1-\omega) \phi-k)-\left(1-\pi_{P}\right)\left(1-\alpha_{P}^{L / H}\right) k}{\left(1-\pi_{P}\right)\left(1-\alpha_{P}^{L / H}\right)+\pi_{P}\left(1-\alpha_{P}^{L / L}\right)}
\end{aligned}
$$


Government Mandated Cost Shifting

$$
\begin{aligned}
\frac{\partial \varphi}{\partial \mu_{R}^{H}} & =-\rho_{3}+r \rho_{0} \frac{\pi_{R}\left(1-\alpha_{R}^{L / L}\right)((1-\omega) \phi-k)-\left(1-\pi_{R}\right)\left(1-\alpha_{R}^{L / H}\right) k}{\left(1-\pi_{R}\right)\left(1-\alpha_{R}^{L / H}\right)+\pi_{R}\left(1-\alpha_{R}^{L / L}\right)} \\
\frac{\partial \varphi}{\partial \mu_{R}^{L}} & =-\rho_{4}+r \rho_{0} \frac{\left(1-\pi_{R}\right) \alpha_{R}^{L / H}((1-\omega) \phi-k)-\pi_{R} \alpha_{R}^{L / L} k}{\left(1-\pi_{R}\right) \alpha_{R}^{L / H}+\pi_{R} \alpha_{R}^{L / L}} \\
\frac{\partial \varphi}{\partial \gamma_{R}} & =-r \frac{\pi_{R} \alpha_{R}^{L / L}}{\left(1-\pi_{R}\right) \alpha_{R}^{L / H}+\pi_{R} \alpha_{R}^{L / L}} U_{1}\left(y_{R}-\gamma_{R}-C^{L^{*}}, \delta\left(\theta^{L}, e^{L}\right)\right) \\
& +\frac{\left(1-\pi_{R}\right) \alpha_{R}^{L / H}}{\left(1-\pi_{R}\right) \alpha_{R}^{L / H}+\pi_{R} \alpha_{R}^{L / L}} U_{1}\left(y_{R}-\gamma_{R}-C^{L^{*}}, \delta\left(\theta^{H}, e^{L}\right)\right) \\
& +\frac{\left(1-\pi_{R}\right)\left(1-\alpha_{R}^{L / H}\right)}{\left(1-\pi_{R}\right)\left(1-\alpha_{R}^{L / H}\right)+\pi_{R}\left(1-\alpha_{R}^{L / L}\right)} U_{1}\left(y_{R}-\gamma_{R}-C^{S^{*}}, \delta\left(\theta^{H}, e^{H}\right)\right) \\
& +\frac{\pi_{R}\left(1-\alpha_{R}^{L / L}\right)}{\left(1-\pi_{R}\right)\left(1-\alpha_{R}^{L / H}\right)+\pi_{R}\left(1-\alpha_{R}^{L / L}\right)} U_{1}\left(y_{R}-\gamma_{R}-C^{S^{*}}, \delta\left(\theta^{L}, e^{H}\right)\right)+r(1-\omega) \rho_{0} \\
\frac{\partial \varphi}{\partial \gamma_{p}} & =(1-r)\left[\frac{\left(1-\pi_{P}\right) \alpha_{P}^{L / H}}{\left(1-\pi_{P}\right) \alpha_{P}^{L / H}+\pi_{P} \alpha_{P}^{L / L}} U_{1}\left(y_{p}+\gamma_{p}-C^{L^{*}}, \delta\left(\theta^{H}, e^{L}\right)\right)\right. \\
& +\frac{\pi_{P} \alpha_{P}^{L / L}}{\left(1-\pi_{P}\right) \alpha_{P}^{L / H}+\pi_{P} \alpha_{P}^{L / L}} U_{1}\left(y_{p}+\gamma_{p}-C^{L^{*}}, \delta\left(\theta^{L}, e^{L}\right)\right) \\
& +\frac{\left(1-\pi_{P}\right)\left(1-\alpha_{P}^{L / H}\right)}{\left(1-\pi_{P}\right)\left(1-\alpha_{P}^{L / H}\right)+\pi_{P}\left(1-\alpha_{P}^{L / L}\right)} U_{1}\left(y_{p}+\gamma_{p}-C^{L^{*}}, \delta\left(\theta^{H}, e^{H}\right)\right) \\
& =B C \quad \pi_{P}\left(1-\alpha_{P}^{L / L}\right) \\
& \left.\frac{\left.\pi_{P}\right)\left(1-\alpha_{P}^{L / H}\right)+\pi_{P}\left(1-\alpha_{P}^{L / L}\right)}{\partial \rho_{0}}\left(y_{p}+\gamma_{p}-C^{L^{*}}, \delta\left(\theta^{L}, e^{H}\right)\right)\right]-(1-r) \rho_{0}
\end{aligned}
$$

\begin{tabular}{|c|c|c|c|}
\hline Kuhn-Tucker Condition & Label & Kuhn-Tucker Condition & Label \\
\hline $\begin{array}{llll}\frac{\partial \varphi}{\partial \mu_{p}^{L}} \leq 0, & \mu_{p}^{L} \geq 0 & \text { and } & \mu_{p}^{L} \frac{\partial \varphi}{\partial \mu_{p}^{L}}=0 \\
\frac{\partial \varphi}{\partial \mu_{p}^{H}} \leq 0, & \mu_{p}^{H} \geq 0 & \text { and } & \mu_{p}^{H} \frac{\partial \varphi}{\partial \mu_{p}^{H}}=0 \\
\frac{\partial \varphi}{\partial \mu_{R}^{H}} \leq 0, & \mu_{R}^{H} \geq 0 & \text { and } & \mu_{p}^{H} \frac{\partial \varphi}{\partial \mu_{R}^{H}}=0\end{array}$ & $\begin{array}{l}(\mathrm{A}-3) \\
(\mathrm{A}-4)\end{array}$ & $\begin{array}{l}\frac{\partial \varphi}{\partial \rho_{3}}=1-\mu_{R}^{H} \geq 0, \quad \rho_{3} \geq 0 \quad \text { and } \quad \rho_{3} \frac{\partial \varphi}{\partial \rho_{3}}=0 \\
\frac{\partial \varphi}{\partial \rho_{4}}=1-\mu_{R}^{L} \geq 0, \quad \rho_{4} \geq 0 \quad \text { and } \quad \rho_{4} \frac{\partial \varphi}{\partial \rho_{4}}=0\end{array}$ & $\begin{array}{l}(\mathrm{A}-8) \\
(\mathrm{A}-9)\end{array}$ \\
\hline
\end{tabular}

Derivatives with respect to the multipliers return the inequality constraints. Because government can always increase the utility of individuals with excess revenue, $\rho_{0}{ }^{*}>0$. The Kuhn-Tucker conditions are given in the table below: 
Government Mandated Cost Shifting

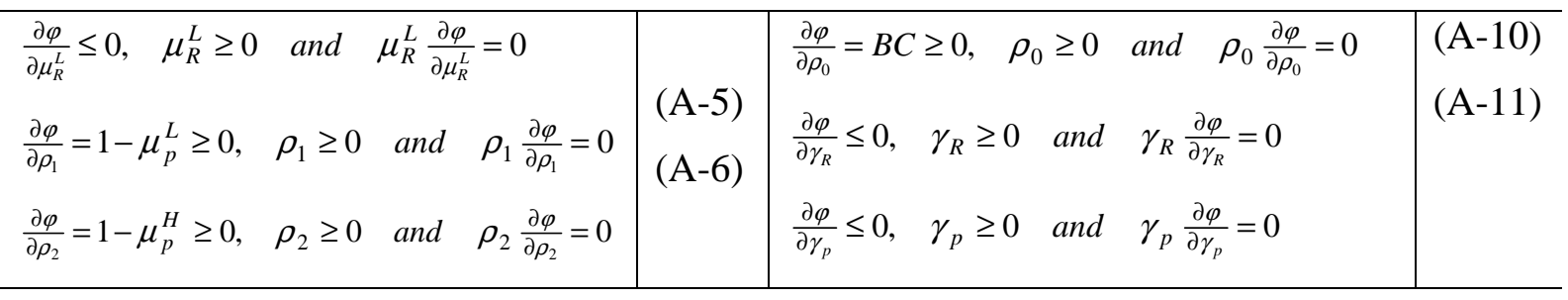

Using the derivatives with respect to $\rho_{0}$ returns the budget constraint.

\subsection{The Physician's Problem}

The physician's problem is to choose their strategies to maximize the sum of (5a) and (5b) in the text. The sum will be referred to as $E V$. The non-negativity constraints are $\alpha_{p}^{L / H} \geq 0, \alpha_{p}^{L / L} \geq 0, \alpha_{R}^{L / H} \geq 0, \alpha_{R}^{L / L} \geq 0$ and the inequality constraints are:

$$
\begin{array}{lll}
1-\alpha_{p}^{H / L} \geq 0 & \text { (IE5) } & 1-\alpha_{R}^{H / L} \geq 0 \\
1-\alpha_{p}^{L / L} \geq 0 & \text { (IE6) } & 1-\alpha_{R}^{L / L} \geq 0
\end{array}
$$

The Physician's problem is to maximize the function:

$$
\xi=E V+\lambda_{1} I E 5+\lambda_{2} I E 6+\lambda_{3} I E 7+\lambda_{4} I E 8
$$

with the choices $\left\{\alpha_{p}^{L / L}, \alpha_{p}^{L / H}, \alpha_{R}{ }^{L / L}, \alpha_{R}{ }^{L / H}\right\}$ taken as given $\left\{\mu_{p}{ }^{L}, \mu_{p}{ }^{H}, \mu_{R}{ }^{L}, \mu_{R}{ }^{H}, \gamma_{p}, \gamma_{R}\right\}$ and $C^{S}$. The partial derivatives are:

$$
\begin{aligned}
& \frac{\partial \xi}{\partial \alpha_{P}^{L / H}}=(1-r)\left(1-\pi_{P}\right)\left[-V\left(C^{L^{*}}, e^{H}\right)+\mu_{P}^{L} V\left(C^{L^{*}}-\phi, e^{L}\right)+\left(1-\mu_{P}^{L}\right) V\left(C^{L^{*}}, e^{L}\right)\right]-\lambda_{1} \\
& \left.\frac{\partial \xi}{\partial \alpha_{P}^{L / L}}=(1-r) \pi_{P}\left[V\left(C^{L}, e^{L}\right)-\mu_{P}^{H} V\left(C^{L}-\phi, e^{H}\right)-\left(1-\mu_{P}^{H}\right) V\left(C^{L}, e^{H}\right)\right)\right]-\lambda_{2} \\
& \frac{\partial \xi}{\partial \alpha_{R}^{L / H}}=r\left(1-\pi_{R}\right)\left[\left(\mu_{R}^{L} V\left(C^{L}-\phi, e^{L}\right)+\left(1-\mu_{R}^{L}\right) V\left(C^{L}, e^{L}\right)\right)-V\left(C^{S}, e^{H}\right)\right]-\lambda_{3} \\
& \left.\frac{\partial \xi}{\partial \alpha_{R}^{L / L}}=r \pi_{R}\left[V\left(C^{L}, e^{L}\right)-\mu_{R}^{H} V\left(C^{S}-\phi, e^{H}\right)-\left(1-\mu_{R}^{H}\right) V\left(C^{S}, e^{H}\right)\right)\right]-\lambda_{4}
\end{aligned}
$$

The derivative with respect to the multipliers returns the inequality constraints. The Kuhn-Tucker conditions are:

\begin{tabular}{l|l|l|l}
\hline Kuhn-Tucker Condition & Label & Kuhn-Tucker Condition & Label \\
\hline
\end{tabular}




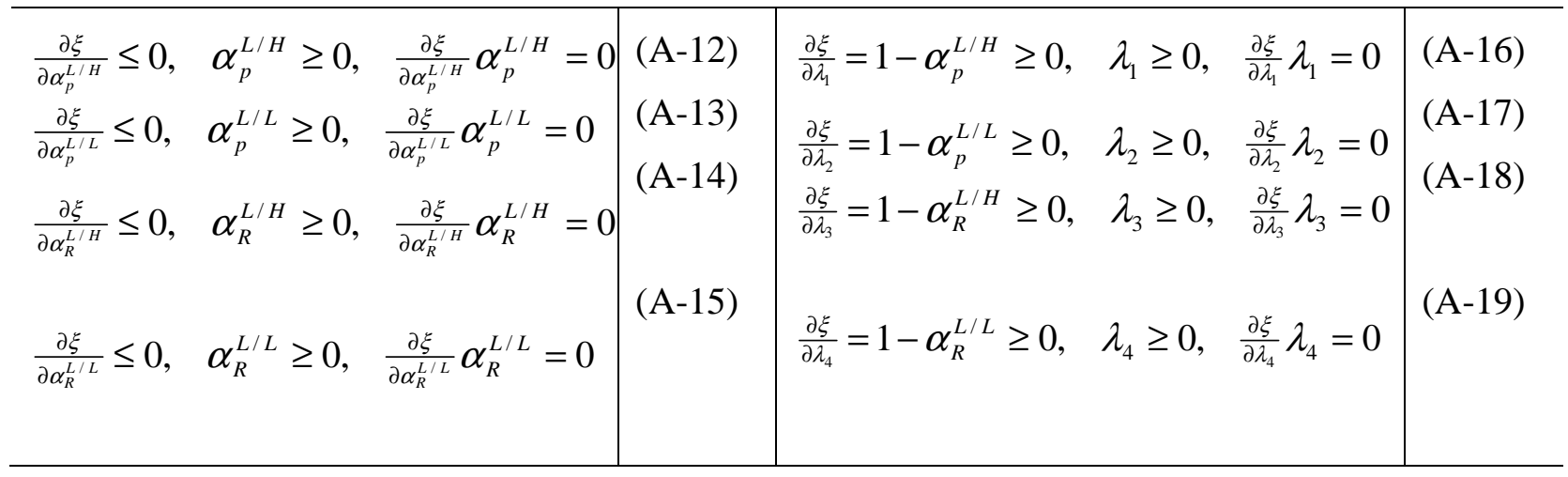

\subsection{Equilibrium}

\section{3.a. Proof of Propositions 1 and $2(k=0)$}

From $\frac{\partial \xi}{\partial \alpha_{P}^{L L}}=\frac{\partial E V}{\partial \alpha_{P}^{L / L}}-\lambda_{2}$ and by the assumption that $V\left(C^{L^{*}}, e^{L}\right)=0$ and $e^{L}<e^{H}$ implies that $\frac{\partial E V}{\partial \alpha_{P}^{L / L}}>0$ regardless of the size of $\mu_{p}{ }^{H}$. Then from the first order condition in (A-13), $\lambda_{2}{ }^{*}>$ 0 . Which from (A-17) implies $\alpha_{p}^{L / L^{*}}=1$. Hence $\alpha_{p}^{L / L^{*}}=1, \lambda_{2}{ }^{*}>0$. From $\frac{\partial \xi}{\partial \alpha_{R}^{L / H}}$ and by the assumption that $V\left(C^{L^{*}}, e^{L}\right)=0$, and $V\left(C^{S^{*}}, e^{H}\right)>0$ and with $\lambda_{3} \geq 0$ then $\frac{\partial \xi}{\partial \alpha_{R}^{L / H}}<0$ regardless of the size of $\mu_{p}^{H}$. Then the last condition in (A-14) implies $\alpha_{R}^{L / H^{*}}=0$. This implies that $\frac{\partial \xi}{\partial \lambda_{3}}>0$ and using $(\mathrm{A}-18): \alpha_{R}{ }^{L / H^{*}}=\mathbf{0}$ and $\lambda_{3}{ }^{*}=0$. With $\alpha_{p}{ }^{L / L^{*}}=1$ and $\frac{\partial \varphi}{\partial \mu_{p}^{H}}=-\rho_{2} \leq 0$ so by (A-2) and (A-6) $\mathbf{1}>\mu_{p}^{H^{*}} \geq \mathbf{0}$ and $\rho_{2}=0$. With $\alpha_{R}{ }^{L / H^{*}}=0$ and $\frac{\partial \varphi}{\partial \mu_{R}^{L}}=-\rho_{4} \leq 0$ so by (A-4) and (A-8) $\mathbf{1}>\boldsymbol{\mu}_{\boldsymbol{R}}^{L^{*}} \geq \mathbf{0}$ and $\rho_{4}=0$.

Let $\alpha_{p}^{L / H^{*}}>0$. First note that $\frac{\partial \varphi}{\partial \mu_{p}^{L}}=\frac{\partial B C}{\partial \mu_{p}^{L}}-\rho_{1}$ and recall that $\alpha_{p}^{L / L^{*}}=1$. Then $\frac{\partial B C}{\partial \mu_{p}^{L}}>0$ so that $\rho_{l}>0$ for $\frac{\partial \varphi}{\partial \mu_{p}^{L}} \leq 0$ that is (A-1). Then from (A-5) $\frac{\partial \varphi}{\partial \rho_{1}}=0$ or $\mu_{p}{ }^{L}=1$. Using $\lambda_{l} \geq 0$ and $\frac{\partial \xi}{\partial \alpha_{P}^{L / H}}=(1-r)\left(1-\pi_{P}\right)\left[-V\left(C^{L^{*}}, e^{H}\right)+\mu_{P}^{L} V\left(C^{L^{*}}-\phi, e^{L}\right)\right]-\lambda_{1}$ we have $\frac{\partial \xi}{\partial \alpha_{P}^{L / H}}<0$, by (1). Then $\alpha_{p}^{L / H^{*}}>0$ and $\frac{\partial \xi}{\partial \alpha_{P}^{L I H}}<0$ are not consistent with the last condition in (A-12). And using (A16), $\alpha_{p}{ }^{L / H^{*}}=\mathbf{0}$ and $\lambda_{l}{ }^{*}=0$. Let $\mu_{p}{ }^{L}=0$, and using $\lambda_{l}{ }^{*}=0$ implies $\frac{\partial \xi}{\partial \alpha_{P}^{L / H}}>0$ which is not consistent with (A-11). So $\mu_{p}{ }^{L}=0$ is not consistent with the Kuhn-Tucker conditions. Given these and $\frac{\partial \xi}{\partial \alpha_{P}^{L / H}} \leq 0$ in (A-12), then $\mu_{P}^{L^{*}} \geq \frac{V\left(C^{L^{*}}, e^{H}\right)}{V\left(C^{L^{*}}-\phi, e^{L}\right)}$. Then from $\mu_{p}^{L^{*}}>0$, $\frac{\partial \varphi}{\partial \mu_{p}^{L}}=0$ from (A-1). From (1), $\mu_{p}^{L^{*}}<1$ and from (A-5) $\rho_{l}{ }^{*}=0$. Therefore $\mathbf{0}<\mu_{p}^{L^{*}} \leq \mathbf{1}$ and $\rho_{l}{ }^{*}=0$. Let $0 \leq \alpha_{R}^{L / L}<1$. Note that $\frac{\partial \varphi}{\partial \mu_{R}^{H}}=\frac{\partial B C}{\partial \mu_{R}^{H}}-\rho_{3}$. Then $\frac{\partial B C}{\partial \mu_{R}^{H}}>0$ so $\rho_{3}>0$ for (A-3) to be satisfied and $\mu_{R}^{H}=1$ from (A-7). Also with $0 \leq \alpha_{R}^{L L}<1$ using (A-19) $\frac{\partial \xi}{\partial \lambda_{4}}>0$ so $\lambda_{4}=0$. However, given these and (2), $\frac{\partial \xi}{\partial \alpha_{R}^{L / L}}=r \pi_{R}\left[-V\left(C^{S}-\phi, e^{H}\right)\right]>0$ and so violates (A-15) so $\leq$ $\alpha_{R}^{L / L}<1$ is not consistent with Kuhn-Tucker conditions. Hence by (A-19) $\left(1-\alpha_{R}^{L / L^{*}}\right)=0$ 


\section{Government Mandated Cost Shifting}

and $\lambda_{4}{ }^{*}=0$. Given these and $\frac{\partial \xi}{\partial \alpha_{R}^{L L}} \leq 0$ in (A-15), so $\mu_{R}^{H^{*}} \geq \frac{V\left(C^{S^{*}}, e^{H}\right)}{V\left(C^{S^{*}}, e^{H}\right)-V\left(C^{S^{*}}-\phi, e^{H}\right)}$ and from (2) and (A-7): $0<\mu_{R}{ }^{H^{*}} \leq 1$ and $\rho_{3}{ }^{*}=0$. This proves equations (6) and (7) in the text.

\section{3.b. Proof of Propositions 3 and 4}

The proofs for $\mu_{R}^{L^{*}}, \mu_{p}^{H^{*}}, \alpha_{R}{ }^{L H^{*}}$ and $\alpha_{R}{ }^{L / L^{*}}$ are the same as when $k=0$ and is not be repeated. The following then are proof for equations (10) and (11) for $\alpha_{R}^{L / L^{*}}$ and $\alpha_{p}^{L / H^{*}}$

Let $\alpha_{p}^{L / H}=0$ then $\frac{\partial \varphi}{\partial \mu_{p}^{L}}<0$ so from (A-1) $\mu_{p}{ }^{L}=0$ and from (A-16) $\frac{\partial \xi}{\partial \lambda_{1}}>0$ so $\lambda_{l}=0$. Given these $\frac{\partial \xi}{\partial \alpha_{p}^{L / H}}>0$ which violates (A-12), so $\alpha_{p}^{L / H}>0$ in equilibrium.

Let $\alpha_{p}^{L / H}=1$. First note that $\frac{\partial \varphi}{\partial \mu_{p}^{L}}=\frac{\partial B C}{\partial \mu_{p}^{L}}-\rho_{1}$ and recall that $\alpha_{p}^{L / L^{*}}=1$. Then using Assumption 3, $\frac{\partial B C}{\partial \mu_{p}^{L}}>0$ so that $\rho_{l}>0$ for $\frac{\partial \varphi}{\partial \mu_{p}^{L}} \leq 0$, that is (A-1). Then from (A-5) $\frac{\partial \varphi}{\partial \rho_{1}}=0$ or $\mu_{p}{ }^{L}=1$. Using $\lambda_{1} \geq 0$ and $\frac{\partial \xi}{\partial \alpha_{P}^{L I H}}=(1-r)\left(1-\pi_{P}\right)\left[V\left(C^{L^{*}}-\phi, e^{L}\right)-V\left(C^{L^{*}}, e^{H}\right)\right]-\lambda_{1}$, I have $\frac{\partial \xi}{\partial \alpha_{P}^{L / H}}<0$, by (1). Then $\alpha_{p}^{L / H}=1$ and $\frac{\partial \xi}{\partial \alpha_{P}^{L / H}}<0$ are not consistent with the last condition in (A-12). And combining (A-16): $\mathbf{0}<\alpha_{p}^{L / H^{*}}<\mathbf{1}$ and $\lambda_{l}{ }^{*}=0$. Given these and $\frac{\partial \xi}{\partial \alpha_{P}^{L / H}}=0$ in (A-12), then $\mu_{p}^{L^{*}}=\frac{V\left(C^{L^{*}}, e^{H}\right)}{V\left(C^{L^{*}}-\phi, e^{L}\right)}$. Using (1) and (A-5): $\mathbf{0}<\mu_{p}^{L^{*}}<1 \rho_{l}{ }^{*}=0$. Then from $\mu_{p}^{L^{*}}>$ $0 \frac{\partial \varphi}{\partial \mu_{p}^{L}}=0$ and using $\alpha_{p}^{L / L^{*}}=1, \alpha_{p}^{L / H^{*}}=\frac{\pi_{p} k}{\left.\left(1-\pi_{p}\right)(1-\omega) \phi-k\right)}$.

Let $\alpha_{R}^{L / L}=0$ then $\frac{\partial B C}{\partial \mu_{R}^{H}}>0$ so $\rho_{3}>0$ for (A-3) to be satisfied and $\mu_{R}^{H}=1$ for (A-7). Also with $\alpha_{R}^{L / L}=0$ using (A-19) $\frac{\partial \xi}{\partial \lambda_{4}}>0$ so $\lambda_{4}=0$. However, given these and (2) $\frac{\partial \xi}{\partial \alpha_{R}^{L / L}}=r \pi_{R}\left[V\left(C^{L^{*}}, e^{L}\right)-V\left(C^{S}-\phi, e^{H}\right)\right]>0$, and so violates $(\mathrm{A}-15)$ so $\alpha_{R}^{L / L}=0$ is not consistent with Kuhn-Tucker conditions.

Let $\alpha_{R}^{L / L}=1$ then $\frac{\partial \varphi}{\partial \mu_{R}^{H}}<0$ and to satisfy (A-3), $\mu_{R}{ }^{H}=0$. Given these, $\frac{\partial \xi}{\partial \alpha_{R}^{L / L}}=-r \pi_{R} V\left(C^{S}, e^{H}\right)-\lambda_{4}<0$, so $\alpha_{R}^{L / L}=1$ violates (A-15) and so is not consistent with the Kuhn-Tucker conditions. Hence (A-19) $\mathbf{0}<\left(\mathbf{1}-\boldsymbol{\alpha}_{R}{ }^{L / L^{*}}\right)<\mathbf{1}$ and $\lambda_{4}{ }^{*}=0$. Given these and $\frac{\partial \xi}{\partial \alpha_{R}^{L / L}}=0$ in (A-15) $\mu_{R}^{H^{*}}=\frac{V\left(C^{S^{*}}, e^{H}\right)}{V\left(C^{S^{*}}, e^{H}\right)-V\left(C^{S^{*}}-\phi, e^{H}\right)}$ Using (2) and (A-7): $0<\mu_{R}^{\boldsymbol{H}^{*}}<\mathbf{1}$ and $\rho_{3}{ }^{*}=0$. With $\mu_{R}^{H^{*}}>0$ then $\frac{\partial \varphi}{\partial \mu_{R}^{H}}=0$ from (A-3). From (A-3) and using $\alpha_{R}^{L / H^{*}}=0$ then: $\left(1-\alpha_{R}^{L / L^{*}}\right)=\frac{\left(1-\pi_{R}\right) k}{\pi_{R}((1-\omega) \phi-k)}$. This proofs equations 11 and 12 .

\section{3.c. Proof of $\gamma_{j}^{*}$}

Using the assumption that $U_{21}=U_{12}=0, \frac{\partial \varphi}{\partial \gamma_{p}}=2 U_{1}\left(y_{p}+\gamma_{p}-C^{L^{*}}\right)-\rho_{0} \leq 0$. The assumption that $U_{1}>0$ implies $\rho_{0}{ }^{*}>0$ for (A-11) to be satisfied. Then from (A-9), $\frac{\partial \varphi}{\partial \rho_{0}}=B C=0$. Given $U_{1}>0, \rho_{0}^{*}>0$ and using the assumption that $U_{21}=U_{12}=0$, define $\omega^{A}$ 


\section{Government Mandated Cost Shifting}

$\frac{\partial \varphi}{\partial \gamma_{R}}=-\left[U_{1}\left(y_{R}-\gamma_{R}-C^{L^{*}}\right)+U_{1}\left(y_{R}-\gamma_{R}-C^{S^{*}}\right)\right]+\left(1-\omega^{A}\right) \rho_{0}=0$ at $\gamma_{R}=0$ so when $\omega \geq \omega^{A} \quad \gamma_{R}^{*}=$

0. Hence $\rho_{0}^{*}=\frac{U_{1}\left(y_{R}-\gamma_{R}-C^{L^{*}}\right)+U_{1}\left(y_{R}-\gamma_{R}-C^{S^{*}}\right)}{\left(1-\omega^{A}\right)}$. With $\alpha_{R}^{L / H^{*}} \geq 0,\left(1-\alpha_{R}^{L / L^{*}}\right) \geq 0, \alpha_{p}^{L / L^{*}}=1, \alpha_{R}^{L / H^{*}}$, $\mu_{p}^{L^{*}}>0, \mu_{R}^{H^{*}}>0 \mu_{p}^{H^{*}}=0, \mu_{R}^{L^{*}}=0$ and $\omega \geq \omega^{A}$ so $\gamma_{R}{ }^{*}=0, B C$ becomes:

$$
-(1-r) \gamma_{P}+(1-r) \mu_{P}^{L} \frac{\left(1-\pi_{R}\right) \alpha_{R}^{L / H}((1-\omega) \phi-k)-\pi_{R} \alpha_{R}^{L / L} k}{\left(1-\pi_{R}\right) \alpha_{R}^{L / H}+\pi_{R} \alpha_{R}^{L L}}+r \mu_{R}^{H} \frac{\pi_{R}\left(1-\alpha_{R}^{L / L}\right)((1-\omega) \phi-k)-\left(1-\pi_{R}\right)\left(1-\alpha_{R}^{L / H}\right) k}{\left(1-\pi_{R}\right)\left(1-\alpha_{R}^{L / H}\right)+\pi_{R}\left(1-\alpha_{R}^{L L}\right)}=0
$$

with each of the last two terms equalling zero and so $\gamma_{p}^{*}=\mathbf{0}$. 